TITLE:

\title{
Ag nanorod arrays tailored for surface-enhanced Raman imaging in the near-infrared region
}

\section{AUTHOR(S):}

Suzuki, Motofumi; Maekita, Wataru; Wada, Yoshinori; Nagai, Koji; Nakajima, Kaoru; Kimura, Kenji; Fukuoka Takao; Mori, Yasushige

\section{CITATION:}

Suzuki, Motofumi ...[et al]. Ag nanorod arrays tailored for surface-enhanced Raman imaging in the near-infrared region. Nanotechnology 2008, 19(26): 265304.

\section{ISSUE DATE:}

2008-05-18

URL:

http://hdl.handle.net/2433/72803

\section{RIGHT:}

Copyright (C) Institute of Physics and IOP Publishing Limited 2008.; This is an author-created, un-copyedited version of an article accepted for publication in Nanotechnology. IOP Publishing Ltd is not responsible for any errors or omissions in this version of the manuscript or any version derived from it. The definitive publisher authenticated version is available online at 10.1088/0957-4484/19/26/265304; この論文は出版社版でありません。引用の際には出版社版をご 確認ご利用ください。;This is not the published version. Please cite only the published version. 


\title{
Ag nanorod arrays tailored for surfaceenhanced Raman imaging in near-infrared region
}

\author{
Motofumi Suzuki ${ }^{1}$, Wataru Maekita ${ }^{1}$, Yoshinori Wada ${ }^{1}$, Koji \\ Nagai $^{1}$, Kaoru Nakajima ${ }^{1}$, Kenji Kimura ${ }^{1}$, Takao Fukuoka ${ }^{2}$ and \\ Yasushige Mori ${ }^{3}$ \\ ${ }^{1}$ Department of Micro Engineering, Kyoto University, Kyoto 606-8501, Japan \\ 2 JST Kyoto Pref. CREATE, Seika, Kyoto 619-0237, Japan \\ 3 Department of Chemical Engineering and Materials Science, Doshisha University, \\ Kyotanabe, Kyoto 610-0394, Japan \\ E-mail: m-snki@me.kyoto-u.ac.jp
}

\begin{abstract}
Using a dynamic oblique angle deposition technique, we demonstrate the direct formation of $\mathrm{Ag}$ nanorods with quasi-parallel major axes on a template layer of oxide having a strongly anisotropic surface morphology. The optical properties of the nanorods are tuned by varying the deposition conditions without any pre- or posttreatment, and the resulting Ag nanorod arrays exhibit high surface-enhanced Raman scattering (SERS) activity. In addition to high macroscopic uniformity over a large area, our nanorod arrays contain a high density of isolated nanorods. Using the optimum Ag nanorod arrays, the SERS imaging of the microdroplets of a rhodamine $6 \mathrm{G}$ solution is successfully demonstrated. The space resolution of the imaging is of the order of at least a few $\mu \mathrm{m}$. These features are suitable for the SERS imaging of biomaterials.
\end{abstract}

PACS numbers: 78.30.-j, 68.55.-a, 81.16.Rf, 78.67.Bf, 73.20.Mf

Submitted to: Nanotechnology 


\section{Introduction}

Surface plasma resonance is well known for its interesting and useful near-field characteristics. In particular, the local field enhancement around resonating metal nanoparticles plays a primary role in enhancing various optical phenomena such as scattering, absorption, and fluorescence [1]. Surface-enhanced Raman spectroscopy [2] (SERS) is expected to be applied to biochemical sensing because of its extremely high sensitivity due to which it can be used for single molecule detection [3]. Besides the development of the molecular imaging technology by tip-enhanced Raman scattering [4], SERS substrates that are used to enhance the sensitivity of conventional Raman spectrosocpy and imaging may be useful for the highly sensitive detection of chemicals present in small amounts and for the imaging of the molecular distribution in microobjects such as cells. In order to significantly enhance Raman scattering, it is important to control both the shape and arrangement of the nanoparticles. Moreover, a high density of isolated nanoparticles in the arrays is crucial to maintain a high space resolution achieved in conventional Raman imaging.

Recently, we have demonstrated the direct formation of noble metal nanorods with quasi-parallel major axes on a template layer of oxide having a strongly anisotropic surface morphology by dynamic oblique deposition (DOD). During the deposition, the deposition angle and in-plane direction of the substrate are varied to control the morphology of the nanorods and template [5]. The resulting nanorod arrays of Ag or Au exhibit high SERS activity $[6,7]$. As compared to the nanostructured SERS substrates produced by using lithographic techniques $[8,9]$, the cost of fabricating large nanorod arrays by our technique can be less and a high density of nanoparticles is obtained [7]. In fact, the morphological and optical properties may be tuned by the deposition conditions without any pre- or posttreatment. In addition, a large number of nanorods exist in laser spots of the order of the diffraction limit $(\sim 1 \mu \mathrm{m})$. This implies that our nanorod arrays may be suitable for enhancing Raman imaging. However, the details of tuning and application to the SERS imaging have not been reported. In this paper, 
we present the results of the tuning of $\mathrm{Ag}$ nanorods for SERS applications and also report a feasibility study on SERS imaging. In order to simulate the SERS imaging of microobjects, microdroplets of a rhodamine 6G (R6G) solution have been prepared on the Ag nanorod arrays and successfully imaged with the SERS peaks of R6G.

\section{Experiments}

Since the detailed procedures for sample preparation have been reported in our previous paper [5], we describe them in brief in this paper. Template layers of $\mathrm{SiO}_{2}$ with an anisotropic surface morphology were prepared by the serial bideposition (SBD) technique [10] on a glass or surface-oxidized Si substrate in a DOD apparatus with a base pressure of $1.3 \times 10^{-4} \mathrm{~Pa}$. A tablet of $\mathrm{SiO}_{2}$ (purity 99.9\%) was evaporated from an electron beam (EB) source placed at a distance of $480 \mathrm{~mm}$ from the substrate under a pressure of $7 \times 10^{-3} \mathrm{~Pa}$. During the $\mathrm{SBD}$, the deposition angle $\alpha_{\mathrm{SiO}_{2}}$ measured from the surface normal was fixed at an angle of $79^{\circ}$, while the azimuthal angle $\phi$ was changed rapidly by $180^{\circ}$ with each deposition of an approximately $20-\mathrm{nm}$-thick layer. After 15 cycles of $\mathrm{SBD}, \mathrm{SiO}_{2}$ layers with an approximate thickness $t_{\mathrm{SiO}_{2}}$ of $600 \mathrm{~nm}$ were obtained. Onto this fabricated template layer, Ag was deposited obliquely. The pressure during the $\mathrm{Ag}$ deposition was $3 \times 10^{-4} \mathrm{~Pa}$, and the vapour flux of $\mathrm{Ag}$ was 0.1$0.2 \mathrm{~nm} / \mathrm{s}$. The deposition angle of $\mathrm{Ag}, \alpha_{\mathrm{Ag}}$, was set at $73^{\circ}$, while the azimuth remained unchanged during the Ag deposition. The amount of deposited Ag, $t_{\mathrm{Ag}}$, was $0.9-21 \mathrm{~nm}$ in average thickness, which was so minute that the Ag layers remained discontinuous on the template. The deposition conditions for the samples are listed in table 1 along with the experimental results that are described in the next section.

The Ag nanoparticles were observed directly by scanning electron microscopy (SEM) without any conductive coatings on the samples that were prepared on the surface-oxidized Si substrate for the morphological characterisation. The samples for the optical and SERS measurements were prepared on a glass substrate. The optical transmittance (absorbance) of the samples was measured using a single-beam 
Table 1. Deposition conditions of samples and experimental results for morphological and optical measurements.

\begin{tabular}{ccccc||cc|cc}
\hline \multicolumn{5}{c||}{ deposition conditions } & \multicolumn{2}{|c|}{ morphology } & \multicolumn{2}{|c}{ absorbance peak } \\
$\mathrm{ID}$ & $\alpha_{\mathrm{SiO}_{2}}$ & $t_{\mathrm{SiO}_{2}}$ & $\alpha_{\mathrm{Ag}}$ & $t_{\mathrm{Ag}}$ & $2 b$ & $a / b$ & $E_{s^{\prime}}$ & $E_{p^{\prime}}$ \\
& $\left(^{\circ}\right)$ & $(\mathrm{nm})$ & $\left(^{\circ}\right)$ & $(\mathrm{nm})$ & $(\mathrm{nm})$ & & $(\mathrm{eV})$ & $(\mathrm{eV})$ \\
\hline $\mathrm{A}$ & 79 & 600 & 73 & 0.9 & 14.5 & 1.3 & - & - \\
$\mathrm{B}$ & 79 & 600 & 73 & 2.3 & 35.7 & 1.3 & 2.6 & 3.3 \\
$\mathrm{C}$ & 79 & 600 & 73 & 3.7 & 25.7 & 2.1 & 2.2 & 3.3 \\
$\mathrm{D}$ & 79 & 600 & 73 & 4.6 & 31.9 & 1.9 & 2.1 & 3.3 \\
$\mathrm{E}$ & 79 & 600 & 73 & 7.4 & 33.9 & 2.6 & 1.9 & 3.2 \\
$\mathrm{~F}$ & 79 & 600 & 73 & 13 & 55.8 & 3.3 & 1.4 & 3.2 \\
$\mathrm{G}$ & 79 & 600 & 73 & 21 & 144.7 & 4.1 & 1.2 & 3.1 \\
\hline
\end{tabular}

spectrophotometer in the wavelength range $300 \mathrm{~nm}<\lambda<1500 \mathrm{~nm}$ at normal incidence. On the basis of the study carried out by Hodgkinson and $\mathrm{Wu}[11,12]$, the polarisation of the incident light was defined as either $p^{\prime}$ - or $s^{\prime}$-polarisation in which an electric field vibrates parallel or perpendicular to the deposition plane of $\mathrm{SiO}_{2}$ and $\mathrm{Ag}$, respectively. Since the nanorods were elongated perpendicular to the deposition plane, as described below, $p^{\prime}$ - and $s^{\prime}$-polarisations corresponded to the polarisations perpendicular and parallel to the nanorods, respectively. In order to avoid confusion with the ordinary definition of the polarisation, which is associated with the plane of incidence of light, we have used the prime symbol to indicate the polarisation associated with the deposition plane in this paper.

Raman spectroscopy was carried out using a near-infrared (IR) confocal Raman microscope (The LabRam 1B, HORIBA Jobin Yvon) at an excitation wavelength of $785 \mathrm{~nm}$ and a power of $15 \mathrm{~mW}$. The microscope had a $60 \times$ objective $(\mathrm{NA}=0.7)$ and a confocal pinhole with a diameter of $1.1 \mathrm{~mm}$. For the optimisation of the nanorods, we performed in situ SERS measurements on the Ag nanorod arrays immersed in an 
aqueous solution of 4,4'-bipyridine (BiPy), as reported in our previous studies [6, 7].

Using the optimised nanorod arrays, we demonstrated the SERS imaging. The optimised Ag nanorods were exposed to a mist of the aqueous solution $(0.5 \mathrm{mmol} / \mathrm{l})$ of R6G produced by an ultrasonic atomiser (HM-303N, HONDA Electronics, Japan), such that the microdroplets of R6G solution were deposited on the nanorod arrays. A laser beam was scanned within an area of $80 \times 80 \mu \mathrm{m}^{2}$, and the Raman spectra were obtained in steps of $2 \mu \mathrm{m}$.

\section{Results and discussion}

\subsection{Morphology}

Figure 1 shows the morphology of an $\mathrm{SiO}_{2}$ template layer. The anisotropic morphologies of the $\mathrm{SiO}_{2}$ template are clearly observed. The SEM image of cross section fractured parallel to the deposition plane of the $\mathrm{SiO}_{2}$ flux shows narrow long columnar structures with small zigzag due to SBD [figure 1(b)]. On the contrary, the SEM image of the cross section fractured perpendicular to the deposition plane of the $\mathrm{SiO}_{2}$ flux shows thick bundles of columnar structures [7]. As a result, the surface shows anisotropic corrugation [figure 1(a)]. The anisotropic morphology of the template is physically self-assembled without the use of any lithographic techniques.

When the vapour is incident obliquely, islands are formed at the early stages of growth and cast long shadows on the surface. Owing to the well-known self-shadowing effect, the deposited atoms cannot adhere to this shadowed region. Due to this selfshadowing effect, oblique columns grow in the direction of the incident vapour beam, as shown in figure 2, if the azimuth of the substrate remains fixed during the deposition. Since the deposited atoms can adhere even to the immediate neighbouring regions around the islands in a direction perpendicular to the deposition plane, the columns tend to be bundled perpendicular to the deposition plane with the help of the so-called fan-out effect [13]. In fact, the anisotropic morphology can be observed even on the 

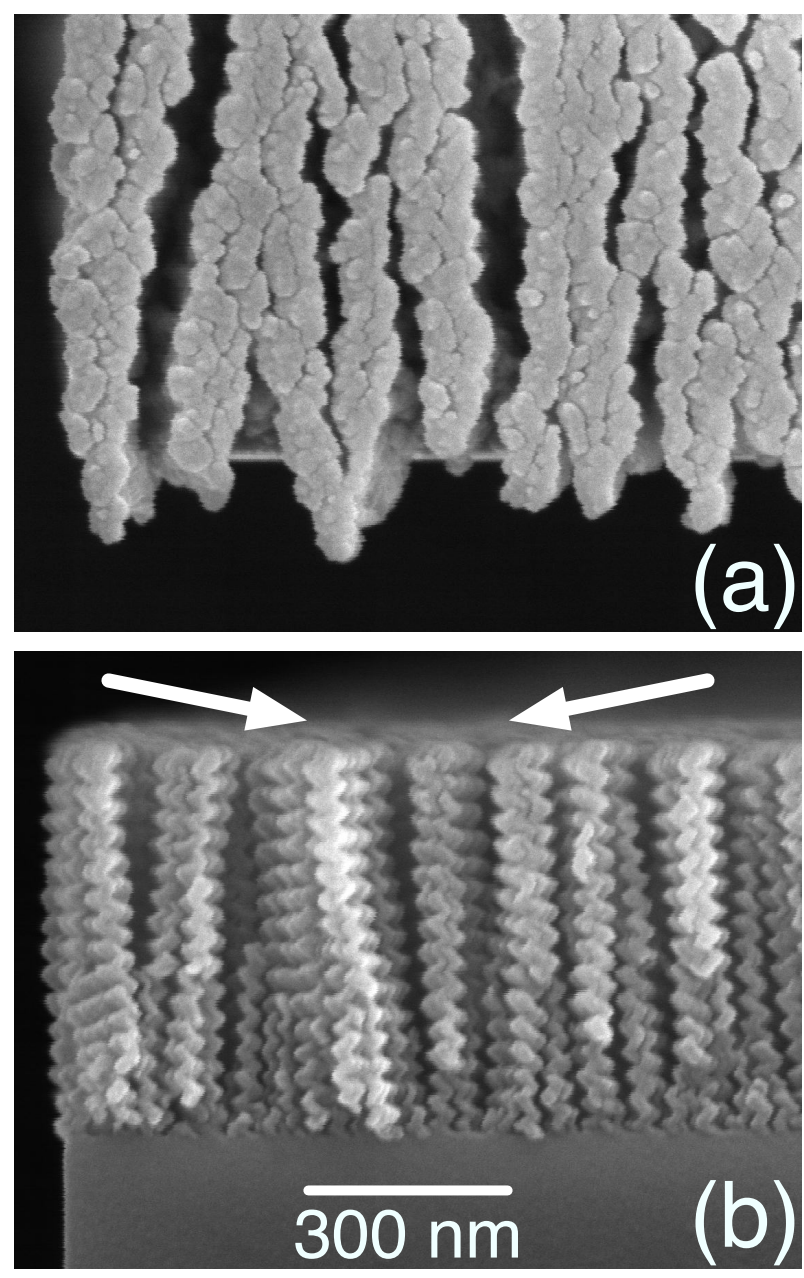

Figure 1. SEM images of an $\mathrm{SiO}_{2}$ template layer: (a) surface and (b) cross section fractured parallel to deposition plane of $\mathrm{SiO}_{2}$. The arrows indicate the deposition directions of $\mathrm{SiO}_{2}$.

surface of the films prepared by simple oblique angle deposition, shown in figure 2(b). In the case of SBD, regions both behind and in front of the columns are shadowed because of the frequent reversions of the substrate such that the degree of anisotropy of the surface morphology is considerably higher than that in the case of simple oblique angle deposition [figures 1(a) and 2(a)]. Thus, we have selected the SBD layer as the template layer and deposited a small amount of $\mathrm{Ag}$ on it obliquely at an angle of $73^{\circ}$.

In order to clarify the shape of the Ag nanoparticles, the samples have been prepared on the surface-oxidized Si substrate and observed by SEM without any conductive coatings. As shown in figure 3, the Ag nanoparticles can be distinguished from the 

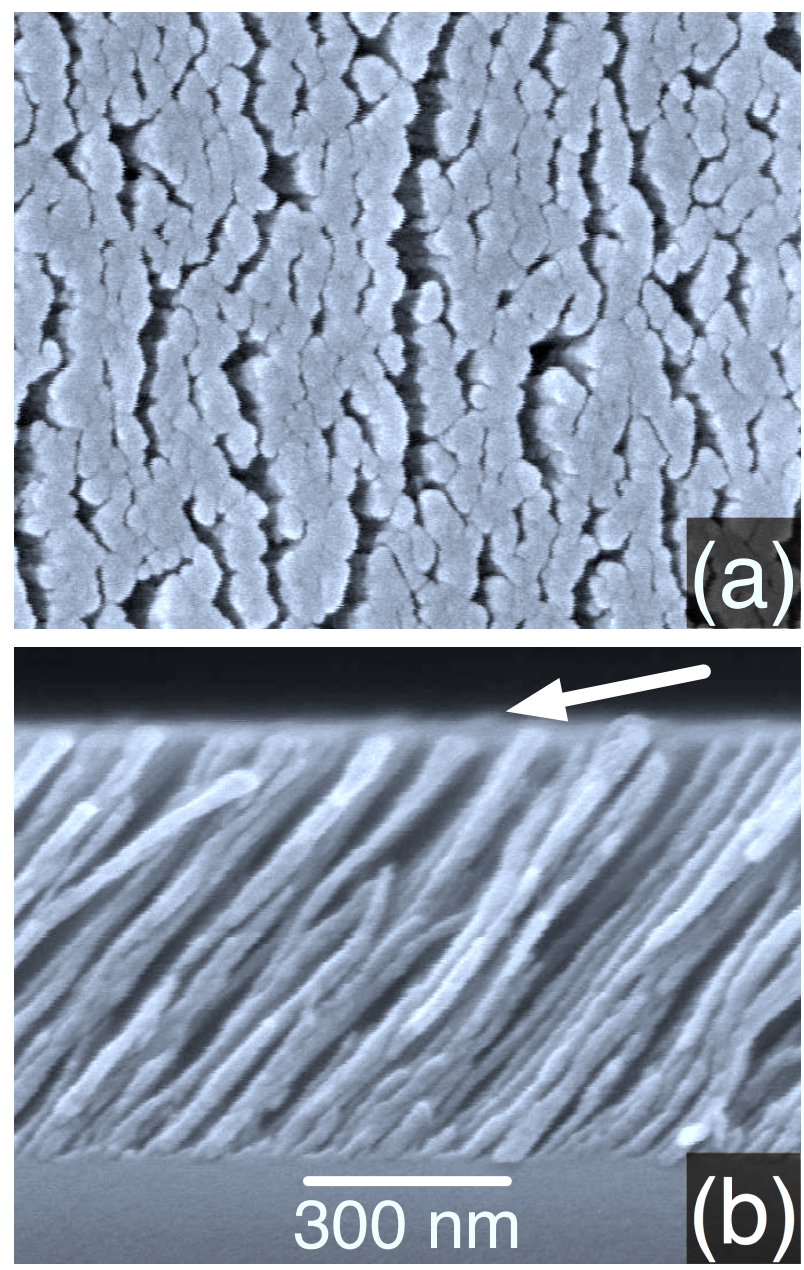

Figure 2. SEM images of a $\mathrm{SiO}_{2}$ film prepared by simple oblique angle deposition at $\alpha_{\mathrm{SiO}_{2}}=79^{\circ}$ without in-plane rotation of substrate: (a) surface and (b) cross section fractured parallel to deposition plane of $\mathrm{SiO}_{2}$. The arrow indicates the deposition direction of $\mathrm{SiO}_{2}$.

$\mathrm{SiO}_{2}$ template in the backscattering electron images. Since the scattering cross section is roughly proportional to $Z^{2}$, where $Z$ is the atomic number of the constituent atoms, the Ag-rich regions appear considerably brighter than the $\mathrm{SiO}_{2}$-rich regions. Many of the white spots observed in figure 3 are attributed to Ag nanoparticles. As indicated by the arrow in figure $3, \mathrm{Ag}$ was deposited in the upper-to-lower direction in these images so that the Ag nanoparticles grow on the tops and slightly toward the upper sides of the elongated columns of the template due to the shadowing effect of the surface corrugation . Clearly, the shape of the Ag nanoparticles depends strongly on the average 

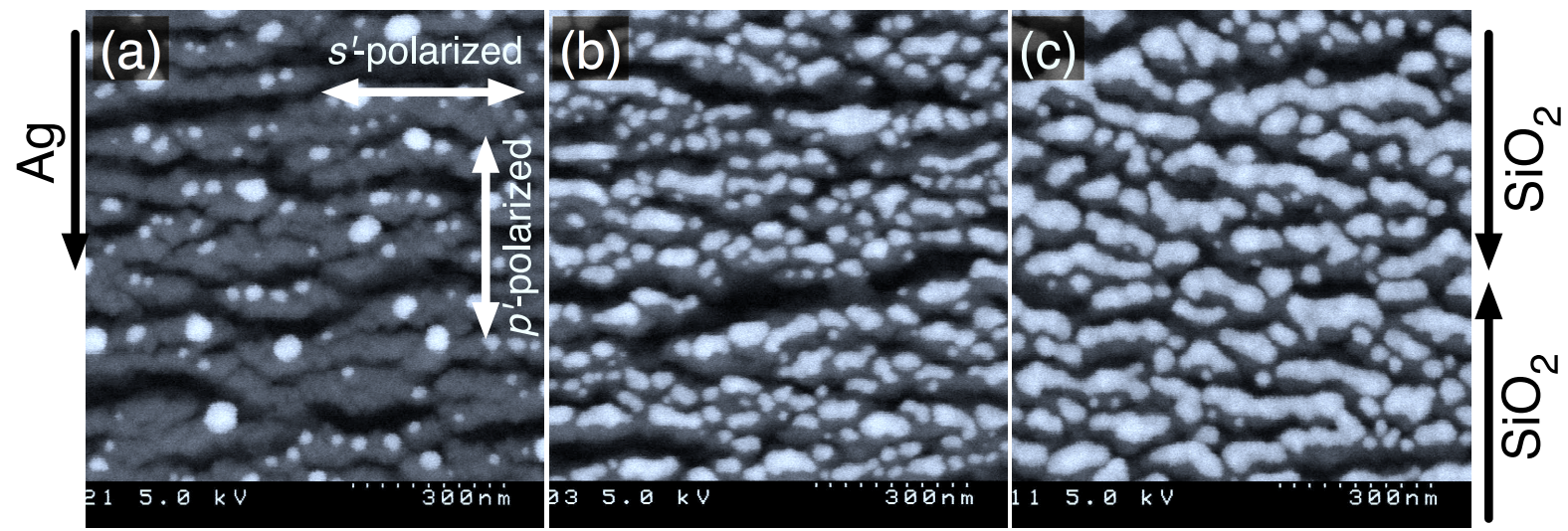

Figure 3. Typical scanning backscattering electron images of samples: (a) B $\left(t_{\mathrm{Ag}}=2.3 \mathrm{~nm}\right),(\mathrm{b}) \mathrm{E}\left(t_{\mathrm{Ag}}=7.4 \mathrm{~nm}\right)$, and $(\mathrm{c}) \mathrm{F}\left(t_{\mathrm{Ag}}=13 \mathrm{~nm}\right)$. The direction of incident $\mathrm{Ag}$ vapour projected on the surface is indicated by the black arrow on the left of the images, and the direction of polarisation of light is indicated by the white arrows. The electric fields of $p^{\prime}$ - and $s^{\prime}$-polarised light vibrate parallel and perpendicular to the deposition plane of the vapour flux, respectively.

thickness of the deposited Ag. The length $(2 a)$ and the width $(2 b)$ of the nanoparticles are summarised in table. 1. For $t_{\mathrm{Ag}}=2.3 \mathrm{~nm}$ (sample B), most of the particles are circular in shape [figure 3(a)]. The average aspect ratio $a / b$ and diameter of the particles are 1.3 and $35 \mathrm{~nm}$, respectively. Although the shape of the nanoparticles is almost isotropic, some nanoparticles are aligned along the edge of the template columns. These aligned particles aggregate into elongated nanoparticles on the samples with larger $t_{\mathrm{Ag}}$, as shown in figures $3(\mathrm{~b})$ and (c). The aspect ratio and width along the minor axis of the nanoparticles of sample $\mathrm{E}\left(t_{\mathrm{Ag}}=7.4 \mathrm{~nm}\right)$ are 2.6 and $35 \mathrm{~nm}$, respectively. The elongated Ag nanoparticles are aligned quasi-parallel to each other. Furthermore, one can observe many gaps between the ends of nanoparticles; the gaps are of the order of a few tens of nm. This appears advantageous for plasmonic sensors such as SERS substrates in enhancing the local electric field. For sample $\mathrm{F}$ with $t_{\mathrm{Ag}}=13 \mathrm{~nm}$, the nanoparticles are more aggregated and become more elongated. However, for the sample F, the number of gaps between the nanoparticles is lesser than that in sample E. These differences in the morphology of the Ag nanoparticles influence the anisotropic plasma resonance and 
SERS properties, as discussed in the following section.

Since the size of the nanoparticles along the normal to the surface, which is estimated from $t_{\mathrm{Ag}}$ and the coverage $\theta$ as $t_{\mathrm{Ag}} / \theta$, is also of the order of a few tens of nm, we call these elongated nanoparticles 'nanorods'.

\subsection{Optical properties}

Figure 4 shows the typical absorbance spectra of $p^{\prime}$ - and $s^{\prime}$-polarised light for samples $\mathrm{B}, \mathrm{E}$, and F. The absorbance of $s^{\prime}$-polarised light increases in the visible or near-IR regions for each sample, while the absorbance of $p^{\prime}$-polarised light increases in the regime of energies higher than that of $s^{\prime}$-polarised light. These spectra consist of a broad absorption band superimposed with small periodic undulation. The broad absorption band is attributed to plasma resonance. On the other hand, the undulation cannot be attributed to the higher order plasma resonance $[14,15]$ since the size distribution of the nanorods is fairly large. The period of these undulations suggests that the interference in the $\mathrm{SiO}_{2}$ template is the origin of the undulation. Thus, we focus our attention on the broad absorption band due to the local plasma resonance. The peak energy of plasmon absorption band of $s^{\prime}$-polarised light, $E_{s^{\prime}}$, for samples $\mathrm{B}, \mathrm{E}$, and $\mathrm{F}$ are 2.6, 1.9, and $1.4 \mathrm{eV}$, respectively, while the peak energy of $p^{\prime}$-polarised light $E_{p^{\prime}}$ is insensitive to the samples. Generally, plasma resonance along the major axis of metal nanoparticles is redshifted due to a decrease in the depolarisation effect, while the resonance along the minor axis is blueshifted slightly $[14,15]$. Therefore, the absorption peaks are attributed to plasma resonance of the free electrons in the Ag nanorods.

The relation between the absorbance peak energies and the aspect ratio of the nanorods is shown in figure 5 . The value of $E_{s^{\prime}}$ decreases with an increase in the aspect ratio, while $E_{p^{\prime}}$ depends weakly on the aspect ratio. Kuwata et al. [16] have proposed a semiempirical formula for the plasma resonance condition for a spheroid as

$$
\left(L+\frac{\varepsilon_{m}}{\varepsilon_{1}+\varepsilon_{m}}\right)+A \varepsilon_{m} x^{2}+B \varepsilon_{m}^{2} x^{4}=0,
$$

where $L$ is the depolarisation factor [15], $\varepsilon_{1}$ is the real part of the relative permittivity of 

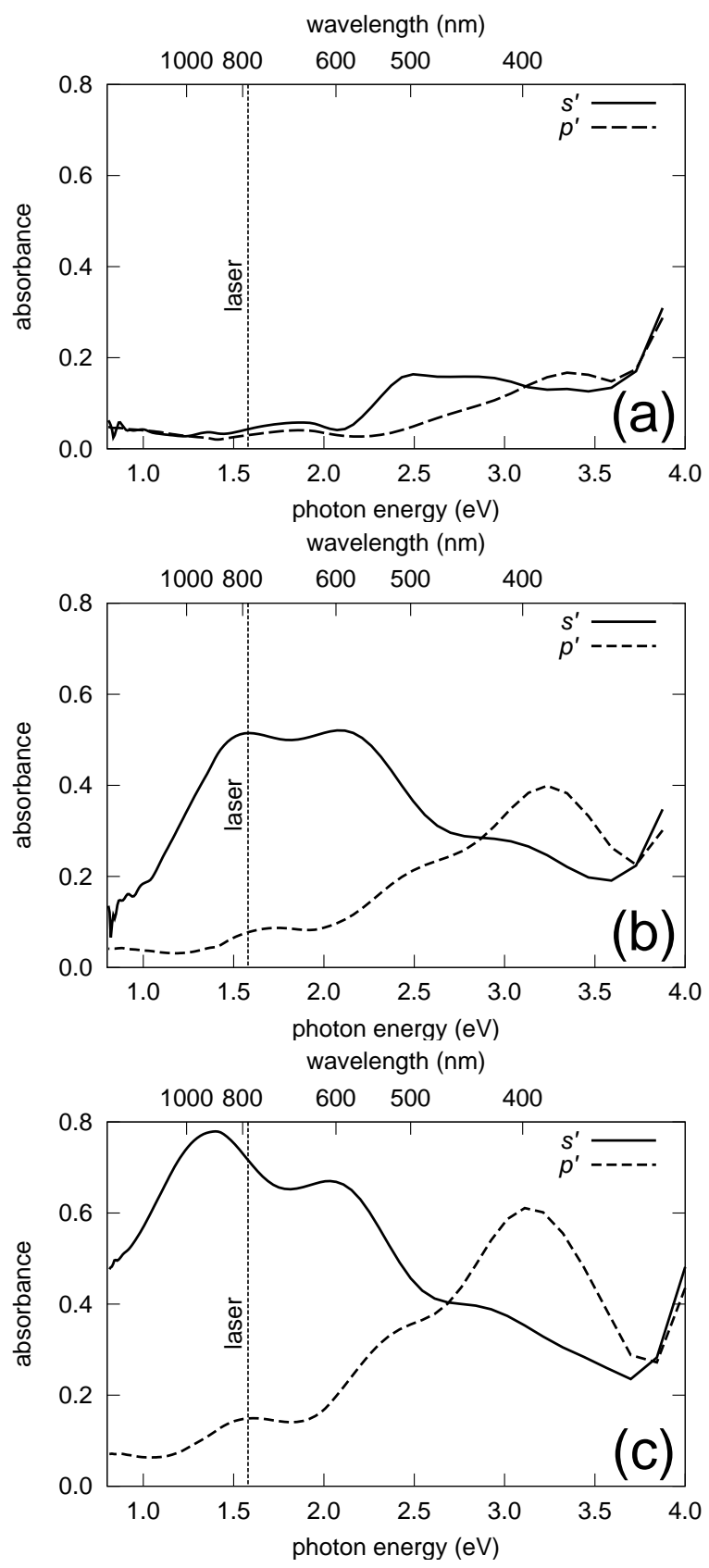

Figure 4. Absorbance spectra as a function of energy of incident light for samples: (a) $\mathrm{B}\left(t_{\mathrm{Ag}}=2.3 \mathrm{~nm}\right)$, (b) $\mathrm{E}\left(t_{\mathrm{Ag}}=7.4 \mathrm{~nm}\right)$, and $(\mathrm{c}) \mathrm{F}\left(t_{\mathrm{Ag}}=13 \mathrm{~nm}\right)$. The absorbance of $s^{\prime}$-polarised light is indicated by the solid line and that of $p^{\prime}$-polarised light by the dashed line. 


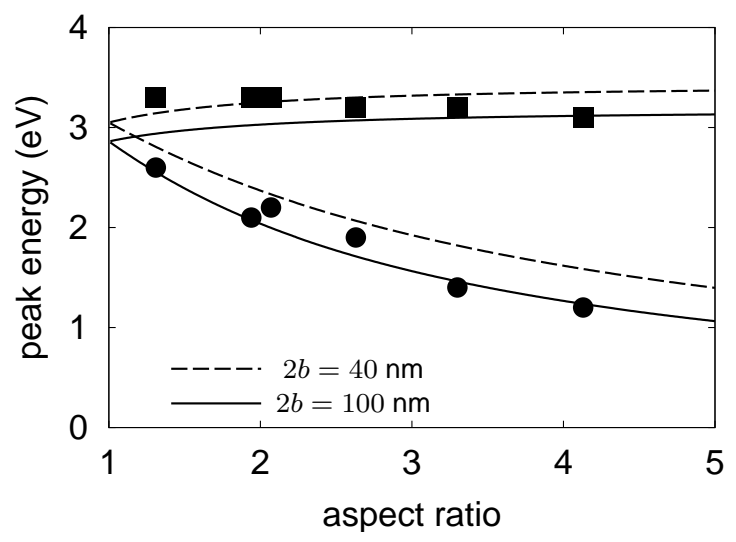

Figure 5. Relation between the aspect ratio of $\mathrm{Ag}$ nanorods and the absorbance peak energies. The measured peak energies of $s^{\prime}$ - and $p^{\prime}$-polarised light are indicated by solid circles and squares, respectively. The lines indicate the plasma resonance energies calculated on the basis of the semiempirical formula proposed by Kuwata et al [16]. The solid lines correspond to the nanorods with 100-nm minor axis and the dashed lines correspond to nanorods with 40-nm minor axis.

the nanorod, $\varepsilon_{m}$ is the relative permittivity of the surrounding medium, and $x=\pi a / \lambda_{0}$. Here, $\lambda_{0}$ is the wavelength of light in vacuum, $A(L)=-0.4865 L-1.046 L^{2}+0.8481 L^{3}$, and $B(L)=0.01909 L+0.1999 L^{2}+0.6077 L^{3}$. Assuming that $\varepsilon_{m}=2.23$ and $\varepsilon_{1}$ is the real part of the relative permittivity of $\mathrm{Ag}$, we have calculated the resonance energy as a function of the aspect ratio for spheroids with minor axis of length $2 b=40$ and $100 \mathrm{~nm}$. The results are plotted in figure 5 along with the measured absorbance peak energies. The plasma resonance energies of $s^{\prime}$ - and $p^{\prime}$-polarised light decrease and increase, respectively, with an increase in the aspect ratio, and the size effect on the resonance energies is not significant. Since the calculated resonance energies agree well with the measured absorbance peak energies, our nanorods can be approximated as spheroids with an average aspect ratio. Since the photon energy of the laser used for Raman spectroscopy is approximately $1.6 \mathrm{eV}$, it is assumed from figure 5 that the nanorods with aspect ratios of 2.5-3.5 enhance Raman scattering significantly. The results of Raman spectroscopy are reported in the next section. 


\subsection{SERS and SERS imaging}

As we have reported in our previous paper, Raman scattering for $s^{\prime}$-polarised light is enhanced significantly as compared to that for $p^{\prime}$-polarised light [6]. In this section, we report the dependence of the SERS intensity on $t_{\mathrm{Ag}}$ and its application to SERS imaging. Figure 6 shows the Raman spectra of the BiPy solution measured on the samples B, E, and F. On sample B, which has a small amount of $\mathrm{Ag}\left(t_{\mathrm{Ag}}=2.3 \mathrm{~nm}\right)$, no enhancement of the Raman scattering is observed. This is consistent with the fact that this sample hardly absorbs the light used in the Raman measurement, as shown in figure 4(a). On the other hand, on samples $\mathrm{E}$ and $\mathrm{F}$, which have large amounts of $\mathrm{Ag}$, the Raman scattering has been enhanced significantly. The clear peaks observed for both $s^{\prime}$ - and $p^{\prime}$-polarised light are characteristics of the SERS of BiPy on noble metal particles [17]. The intensity for $s^{\prime}$-polarised light is considerably higher than that for $p^{\prime}$-polarised light, as reported in our previous paper [6]. In addition, it is clear that the SERS intensity depends on $t_{\mathrm{Ag}}$.

In order to clarify the relation between $t_{\mathrm{Ag}}$ and the SERS intensity, the integrated intensity of the ring in-plane deformation mode of $\mathrm{BiPy}$ around $1014 \mathrm{~cm}^{-1}$, where the linear background is subtracted from the scattering intensity, is plotted as a function of $t_{\mathrm{Ag}}$, as shown in figure 7 . The SERS intensity increases significantly at $t_{\mathrm{Ag}} \sim 4 \mathrm{~nm}$. The intensity for $s^{\prime}$-polarised light reaches a maximum at $t_{\mathrm{Ag}} \sim 8 \mathrm{~nm}$, while that for $p^{\prime}$-polarised light increases gradually with $t_{\mathrm{Ag}}$. Over the entire range of $t_{\mathrm{Ag}}$ employed in this study, the intensity for $s^{\prime}$-polarised light is higher than that for $p^{\prime}$-polarised light, although their contrast decreases with an increase in $t_{\mathrm{Ag}}$. From the discussion in the previous section, significant enhancement of the local electric field is expected when $s^{\prime}$-polarised light is incident on samples $\mathrm{E}$ and $\mathrm{F}$, which exhibit plasma resonance near the wavelength of the light used in Raman spectroscopy. Remarkably, not only samples $\mathrm{E}\left(t_{\mathrm{Ag}}=7.4 \mathrm{~nm}\right)$ and $\mathrm{F}\left(t_{\mathrm{Ag}}=13 \mathrm{~nm}\right)$ but all the samples with $t_{\mathrm{Ag}} \geq 3.7 \mathrm{~nm}$ exhibit rather strong SERS. The gaps of the in-line aligned Ag nanorods may influence the dependence of the SERS intensity on $t_{\mathrm{Ag}}$. Nevertheless, the Ag nanorod arrays with 

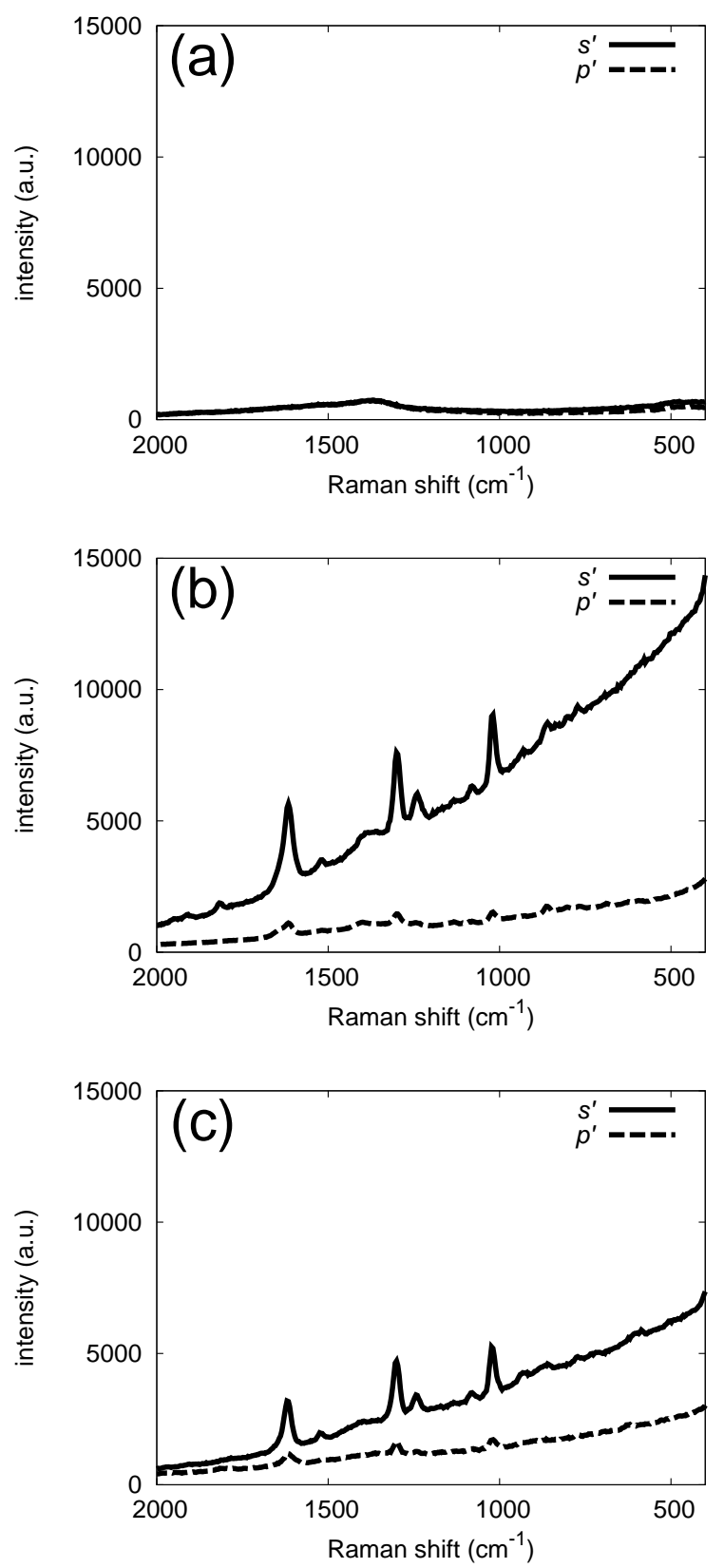

Figure 6. Polarisation-dependent Raman spectra of BiPy solution (1 mmol/l) measured on samples: (a) B $\left(t_{\mathrm{Ag}}=2.3 \mathrm{~nm}\right),(\mathrm{b}) \mathrm{E}\left(t_{\mathrm{Ag}}=7.4 \mathrm{~nm}\right)$, and (c) $\mathrm{F}\left(t_{\mathrm{Ag}}=13\right.$ $\mathrm{nm})$. The excitation laser beam was polarised by a commercial polaroid film with an extinction ratio of 170 at $\lambda=785 \mathrm{~nm}$. 


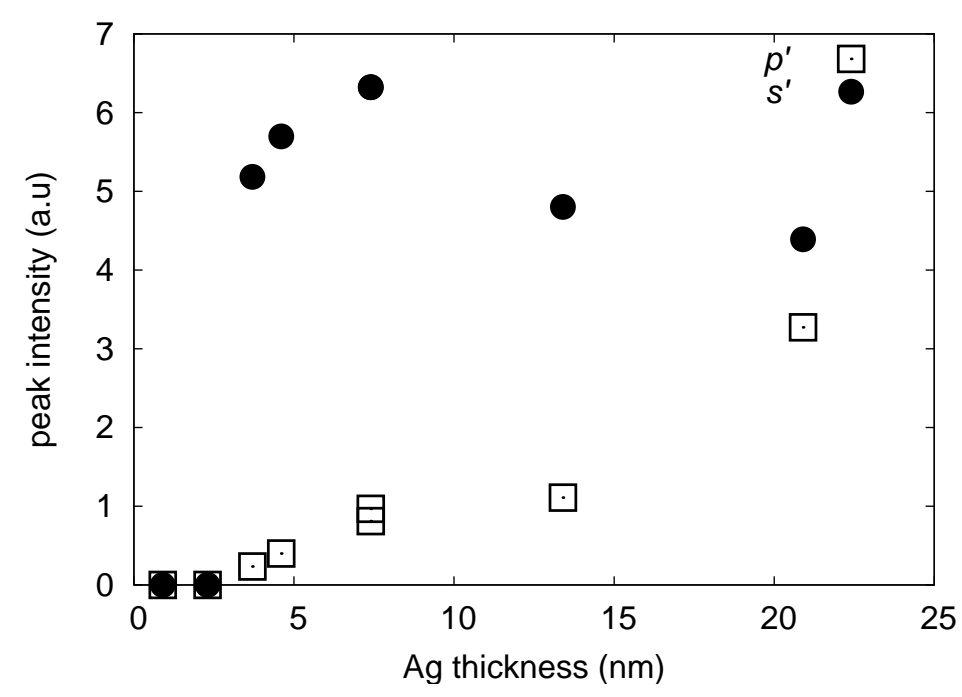

Figure 7. SERS peak intensity at $1014 \mathrm{~cm}^{-1}$ as a function of $t_{\mathrm{Ag}}$. The intensities for $s^{\prime}$ - and $p^{\prime}$-polarised light are indicated by solid circles and open squares, respectively. The intensity is normalised by the value for the samples prepared under condition $\mathrm{E}$ mentioned in table 1.

$t_{\mathrm{Ag}} \sim 8 \mathrm{~nm}$ are suitable for practical SERS sensors.

In order to confirm whether SERS imaging of microobjects is possible by using our nanorod arrays, we deposited microdroplets of the R6G solution on the nanorod arrays prepared under condition E mentioned in table 1. Figure 8(b) shows the optical micrograph of a R6G-deposited surface of the nanorod arrays. The R6G droplets with diameters ranging from a few $\mu \mathrm{m}$ to $\sim 20 \mu \mathrm{m}$ are recognised as dark spots. Figure 8(a) indicates the SERS spectrum measured at a point on a R6G droplet. The peaks observed at $609,788,1184,1311,1361$, and $1509 \mathrm{~cm}^{-1}$ are consistent with those reported as the SERS of R6G $[18,19]$. In order to obtain the SERS images, the region framed by the black square in figure $8(\mathrm{~b})$ was scanned in steps of $2 \mu \mathrm{m}$, and the Raman spectrum was measured at each point by taking a period of $1 \mathrm{~s}$. The SERS images were mapped with the SERS peaks of R6G [figures 8(c), (d), and (f)-(h)]. For comparison, an image was also obtained by using a non-R6G-related intensity around $880 \mathrm{~cm}^{-1}$, as shown in figure 8(e). Clearly, the images created from the peaks of R6G agree well with the optical micrograph, while no significant contrast is observed in the image obtained 


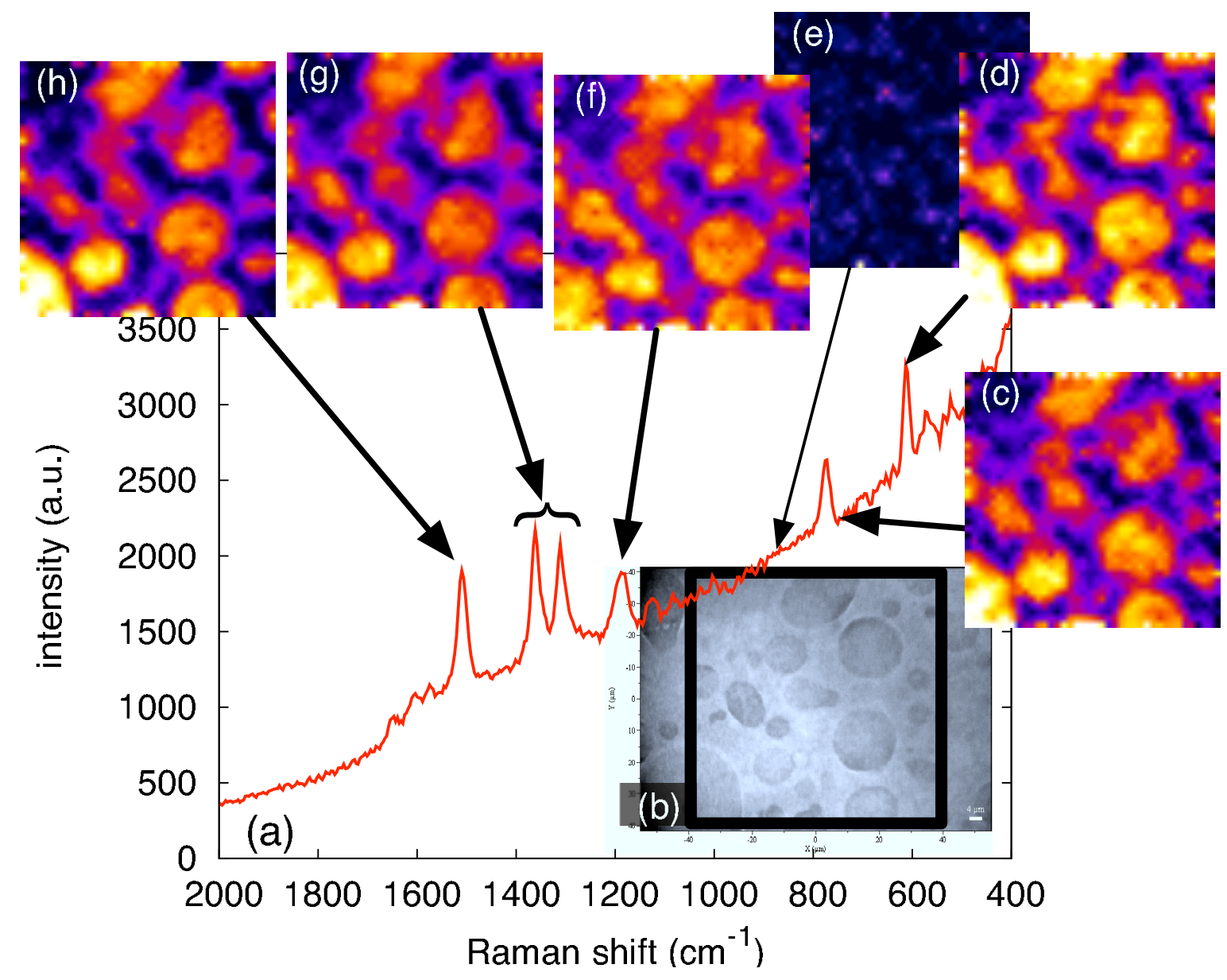

Figure 8. (a) SERS spectrum measured on a R6G droplet, (b) optical micrograph of the R6G-deposited surface of Ag nanorod arrays, and SERS images mapped by Raman intensity around (c) $768 \mathrm{~cm}^{-1}$, (d) $609 \mathrm{~cm}^{-1}$, (e) $880 \mathrm{~cm}^{-1}$, (f) $1184 \mathrm{~cm}^{-1}$, (g) 1336 $\mathrm{cm}^{-1}$ and (h) $1509 \mathrm{~cm}^{-1}$. The bright colours correspond to strong SERS.

from the non-R6G-related scattering. Thus, we have successfully carried out the SERS imaging of the microdroplets of R6G. Comparing the optical and SERS images, we can confirm that the space resolution of SERS imaging in this study is of the order of at least a few $\mu \mathrm{m}$. This high space resolution will enable SERS imaging of biological subjects such as cells, bacteria, and viruses in the near future.

\section{Conclusion}

We have fabricated Ag nanorod arrays tailored for application to near-IR SERS. The anisotropic morphology of a template of $\mathrm{SiO}_{2}$ is physically self-assembled by SBD 
without the use of any lithographic techniques. By depositing Ag on the template obliquely, Ag particles grow on top of the elongated columns of the template leading to the formation of Ag nanorods. The morphology of the Ag nanorods changes systematically as a function of the amount of the deposited Ag. The nanorods exhibit anisotropic optical absorption due to plasma resonance and the absorbance peak energies also change systematically depending on the amount of deposited Ag. The behaviour of the absorbance peak energies has been clarified in terms of the dependence of the plasma resonance on the aspect ratio of the nanorods. The optimum SERS properties are obtained for the nanorods that resonate near the energy of the laser used in Raman spectroscopy. Using the optimum nanorods, the SERS imaging of microdroplets of a R6G solution has been successfully demonstrated. The space resolution of imaging is of the order of at least a few $\mu \mathrm{m}$. Thus, the Ag nanorods created by DOD are promising candidates for the SERS imaging of biological subjects such as cells, bacteria, and viruses.

\section{Acknowledgments}

We are grateful to Sadamu Kinoshita for the SEM observations. This work was supported by The 21st Century COE Program 'Center of Excellence for Research and Education on Complex Functional Mechanical Systems', Grant-in-Aid for Scientific Research (B), 17310073, 2005 by the Ministry of Education, Culture, Sports, Science and Technology, Japan and Nippon Sheet Glass Foundation for Materials Science \& Engineering. 


\section{References}

[1] Wang D S, Chew H and Kerker M 1980 Appl. Opt. 19 2256-2257

[2] Jeanmaire D L and Van Duyne R P 1977 J. Electroanal. Chem. 84 1-20

[3] Kneipp K, Wang Y, Kneipp H, Perelman L T, Itzkan I, Dasari R R and Feld M S 1997 Physical Review Letters $\mathbf{7 8} 1667$

[4] Hayazawa N, Inouye Y, Sekkat Z and Kawata S 2000 Optics Communications 183 333-336

[5] Suzuki M, Maekita W, Kishimoto K, Teramura S, Nakajima K, Kimura K and Taga Y 2005 Jpn. J. Appl. Phys. 44 L193-L195

[6] Suzuki M, Maekita W, Wada Y, Nakajima K, Kimura K, Fukuoka T and Mori Y 2006 Appl. Phys. Lett. 88203121

[7] Suzuki M, Nakajima K, Kimura K, Fukuoka T and Mori Y 2007 Analytical Sciences 23829

[8] Hulteen J C, Treichel D A, Smith M T, Duval M L, Jensen T R and Van Duyne R P 1999 J. Phys. Chem. B 103 3854-3863

[9] Perney N M B, Baumberg J J, Zoorob M E, Charlton M D B, Mahnkopf S and Netti C M 2006 Optics Express 14 847-857

[10] Hodgkinson I J and Wu Q 1999 Appl. Opt. 38 3621-3625

[11] Wu Q H and Hodgkinson I 1994 Journal of Optics 25 43-49

[12] Hodgkinson I J and Wu Q 1997 Birefringent Thin Films and Polarizing Elements (Singapore: World Scientific) ISBN 981-02-2906-2

[13] Lu T M, Ye D X, Karabacak T and Wang G C 2005 Mater. Res. Soc. Symp. Proc. "Kinetics-Driven Nanopatterning on Surfaces" (Mater. Res. Soc. Symp. Proc. vol 849) ed Chason E, Gilmer G H, Huang $\mathrm{H}$ and Wang E (Warrendale, PA) pp 13-18

[14] van de Hulst H C 1981 Light scattering by small particles (New York: Dover)

[15] Bohren C F and Huffman D R 1983 Absorption and Scattering of Light by Small Particles (New York: Wiley)

[16] Kuwata H, Tamarua H, Esumi K and Miyano K 2003 Appl. Phys. Lett. 83 4625-4627

[17] Joo S W 2004 Vib. Spectrosc. 34 269-273

[18] Majoube M and Henry M 1991 Spectrochimica Acta Part A: Molecular Spectroscopy 47 1459-1466

[19] Kneipp K, Roth E, Engert C and Kiefer W 1993 Chemical Physics Letters 207 450-454 